specific qualities traditionally associated with the middle classes were tending to be modified or even to disappear.

The conclusions adopted as a result of the discussions on the three major aspects of the problem showed that no agreement on the definition of the term ' middle class' had been reached; that the existence of middle classes in the tropical and sub-tropical world was not an entirely new phenomenon, but that in recent years there had been a marked increase in the number of persons who might be described as belonging to the middle classes; that a spirit of independence is an outstanding characteristic of the middle classes, and that this has led them to play a major role in the political evolution of their countries. Other conclusions emphasized the valuable part that the middle classes can play in economic development, and that the diversity and heterogeneity of the groups composing a 'middle class' were qualities of value for the development of modern societies, and should be encouraged. Reference was made to the need for further study of this subject.a

\title{
Création d'un Séminaire de Sciences Humaines
}

FrAppé par l'isolement des chercheurs français dans le domaine des Sciences Humaines, un groupe du Musée de l'Homme représentant les tendances les plus diverses vient d'organiser un séminaire indépendant où sont exposées les recherches en cours et confrontées les différentes méthodes.

Le s Décembre 1955, après un exposé de J. Rouch (C.N.R.S.), une large discussion s'ouvrit. Il fut décidé que les réunions du séminaire comporteraient: des exposés de chercheurs concernant le sujet de leurs travaux et les méthodes qu'ils emploient (l'exposé des résultats restant le fait des conférences aux sociétés déjà existantes). Ces exposés, courts, seraient suivis de discussions; des rapports préliminaires sur les missions sur le terrain, avant le dépouillement des résultats de ces missions; des exposés de chercheurs étrangers de passage; des nouvelles relatives aux missions sur le terrain; des exposés de résolutions de Congrès, etc. ... .

C. Lévi-Strauss (École Pratique des Hautes Études) a ouvert dès Décembre la série des exposés en posant la question suivante: 'Les organisations dualistes existent-elles ?' Aux réunions suivantes C. Tardits (O.R.S.T.O.M.) a exposé les méthodes qu'il a utilisées pour étudier l'évolution économique d'une société à structure patrilinéaire dans le Sud Dahomey, et D. Paulme (Musée de l'Homme) quelques points d'une enquête au pays Baga. J. Rouch a fait une critique des méthodes d'enquête classiques à propos d'une étude des migrations en Gold Coast. Aux prochaines réunions L. Bernot (C.N.R.S.) et P. Reichlen (Musée de l'Homme) parleront de problèmes de classification à l'intérieur des Sciences Sociales; S. Dreyfus-Roche (C.N.R.S.) fera un exposé sur sa récente enquête chez des Indiens du Brésil et $\mathrm{H}$. Lehmann (Musée de l'Homme) abordera certains problèmes d'archéologie guatémaltèque.

Les réunions du Séminaire des Sciences Humaines ont lieu deux fois par mois au Musée de l'Homme.

\section{Colloque colonial sur l'Economie indigène}

Sous les auspices de l'Institut de Sociologie Solvay s'est réuni à Bruxelles, du 9 au I 3 janvier 1956, un groupe d'économistes auxquels s'étaient joints des ethnologues. Ce colloque international qui groupait des spécialistes anglais, belges, congolais, hollandais, italiens, français et portugais avait pour objet 'l'étude des moyens susceptibles de promouvoir l'économie indigène dans les pays dépendants et plus spécialement au Congo Belge et au Ruanda-Urundi'. 